\title{
Discussion: Field verification of bed-mounted ADV meters
}

\author{
N. Mclntyre and M. Marshall
}

\author{
Contribution by J. R. Blake and J. C. Packman, Centre for Ecology \\ \& Hydrology, Wallingford, UK \\ McIntyre and Marshall evaluated the accuracy of continuous- \\ signal acoustic Doppler velocity (ADV) meters for measuring \\ stream flow (in this case the Unidata 'Starflow' instrument) by \\ comparison against flows measured with an impeller meter. For \\ very low flows ( $\leqslant 0.02 \mathrm{~m}^{3} / \mathrm{s}$ ) the Starflow accuracy was found \\ to be poor, while for higher flows it ranged from poor to good, \\ improving with calibration, and better for concrete than \\ natural channel sections.
}

At the Centre for Ecology \& Hydrology (CEH) upwards of 50 Starflow (and other ADV) meters have been successfully used at many sites where conventional gauging structures were impractical (sewers, culverts and natural channels in Bracknell, Bradford, Witney, Banbury, Fleet, Otmoor, Wytham, India, Bangladesh and Pakistan) (Blake and Packman, 2008; Water Cycle Management for new developments, 2008; Old et al., 2002; Packman and Hewitt, 1998, 2000). Such check gauging and laboratory testing as has been carried out at CEH has produced considerable confidence in their use, provided suitable instrument positioning, configuration and data processing procedures are adopted. McIntyre and Marshall's findings may not be applicable to ADV monitoring in general or indeed to Starflow instruments used carefully. As ever, proper site selection and field work procedures are crucial to data reliability. The following may have affected their results.

\section{INSTRUMENT POSITIONING}

ADV meters work best where the flow profile is undisturbed and near normal depth. Sites near bends and sudden changes of depth should be avoided. With depth measured directly above the instrument sensor, but velocity some distance up- or downstream (along the inclined path of the ultrasonic beam), any change in depth between these zones will obviously affect the results. As the authors have found, culverts and particularly pipes that focus flow back to the centre, generally give better results. Natural sections often show uneven velocity distributions, with back eddies occurring at low flows. Six of the authors' nine Starflow instruments were positioned with the velocity sensor pointing downstream to reduce damage by storm debris. The authors note that in theory orientation does not affect performance (Vermeyen, 2000). However, the Starflow manual (Unidata, 2000) states that when pointing the sensor downstream, 'in some channels the sensor body may disturb the velocity distribution unacceptably' (p. 23). As discussed below, this will increase noise in the velocity signal, reducing the ability of the instrument to record a valid median velocity. The present authors' experience is that more reliable velocity readings are acquired with the instrument pointing upstream.

Moreover, downstream orientation is more susceptible to the effect of any change in channel characteristics immediately downstream. SF5 (Figure 4) and potentially SF4 and SF6 are located at the downstream end of culverts (as recommended on p. 22 of the Starflow manual (Unidata, 2000) to minimise upstream flow turbulence), however these sites (and the upstream-facing SF3) have a hydraulic drop at the culvert/pipe outfall. Pointing downstream at such locations should be avoided as the measured velocity distribution, and hence recorded median value, may be skewed by spurious lower downstream velocities (see p. 94 of the Starflow manual (Unidata, 2000)). This may explain the large $\alpha$ value at site SF5. Furthermore, monitoring in a hydraulic drop drawdown zone should be avoided (Water Research Centre, 1987) as the variance between depth and velocity measurement locations (discussed above) means that calculated discharges will generally be higher than expected if pointing downstream and lower than expected if pointing upstream. When monitoring in culverts or pipes, it would be advisable to orientate the instrument upstream and, if practical and safe, with the sensing head some distance upstream of the outlet/outfall, perhaps by two to four times the pipe diameter (the sensor-to-manhole distance suggested in ADV sewer monitoring guidance) (Water Research Centre, 1987).

The authors noted from plots of depth-velocity (Figure 5) that the relationship for SF1 changed over time and became more scattered after July 2006, interpreting that the velocity distribution across the channel cross-section changed. The scatter might also be attributed to the instrument acquiring velocity readings from slower-moving bedload targets, which have been newly mobilised by the event(s) that initiated the channel bank erosion. It is possible to infer much information about site hydraulic behaviour from depth-velocity scatter plots (ADS, 2008), aiding evaluation of instrument positioning.

\section{INSTRUMENT CONFIGURATION}

Figure 5 shows that SF2 (and to a lesser extent SF4) exhibits spurious high-velocity readings at low depths. These are caused by signal noise at low actual velocities, although a dynamic 
signal filter (see p. 101 of the Starflow manual (Unidata, 2000)) or a post-processing methodology (Blake and Packman, 2008) can be used to reduce this effect. As the authors do not appear to have activated the Starflow dynamic filter in this study, their conclusions regarding the accuracy of the instrument, particularly at low flows when spurious high velocities are likely to occur, should be viewed with caution.

The authors have used Starflow instruments with factory settings (Unidata, 2000) (1 min scan rate, 15 min logging, $2 \mathrm{~s}$ maximum sampling period) except for 'maximum samples' ('samples' being the processed Doppler signal returns), which has been set to 500 (the factory default is 200). If the instrument cannot acquire the minimum number of samples (factory setting 20) within the sampling period, the default behaviour is to use the last good velocity reading (which may or may not be representative of the actual velocity, especially if the last good reading was acquired some time previously). This is most likely under low flow conditions for 'clean' flows, when there may be too few scattering particles passing by the instrument to provide a reliable median velocity (Unidata, 2000). However, this situation can be mitigated by modifying the instrument firmware settings to increase the sampling period (Blake and Packman, 2008). The present authors' experience is that a $7 \mathrm{~s}$ maximum sampling period is possible with 12 Ah batteries, four seconds with 7 Ah batteries (for 1 or 2 min combined scan and logging intervals). Using this configuration improves the ability of the instrument to measure low flows.

The instruments have been configured to scan for velocity every minute but then record only the average value each $15 \mathrm{~min}$. As mentioned above, if there is excessive noise in the sampled velocities (defined by the 'Quartile' setting, default 100\%) or if there are too few samples, the default behaviour is to repeat the last known good velocity. Therefore, particularly for low flows, the $15 \mathrm{~min}$ average calculation may well have included such unreliable repeated velocities. This may explain some of the divergence between ADV logged and manually gauged discharges. The current authors would recommend that raw velocity and depth readings are logged each scan interval, allowing subsequent post-processing to identify and correct velocity measurement errors (Blake and Packman, 2008). The Starflow has sufficient memory to record 41 days of raw velocity and depth data at a $2 \mathrm{~min}$ scan/log interval. Smoothing of the velocities could then be applied as part of the standard data processing procedure. Before calculating flows for calibration the current authors would also recommend adjustment of the instrument depth measurement. First, the height of the instrument ' 0 depth reference' point (see p. 26 of the Starflow manual (Unidata, 2000)) above the pipe invert/ channel bed must be added to the logged depths. Second, the logged depths should be adjusted to account for any pressure transducer bias by adding an offset value to the recorded depths (the offset is the manually measured depth of water above the reference point minus the instrument recorded depth; it should be measured/calculated at regular intervals as it may vary over time, although measurement may be difficult for rapidly varying flows). This instrument configuration, with dynamic signal filter activated, increased maximum sampling period and logging raw velocity and depth values with subsequent velocity error correction and depth adjustment, improves the ability of the instrument to measure low flows.

\section{Authors' reply}

Staff at the Centre for Ecology \& Hydrology (CEH) are recognised as world-leading experts on application of ADV technology to flow measurement. During the design and installation of the hydrometry programme at Pontbren, the authors consulted appropriate CEH staff, who have experience using ADVs in steep upland catchments. The tone of the response by Blake and Packman to the present authors' paper is that the $\mathrm{ADV}$ meters at Pontbren have underperformed. On the contrary, the performances documented in the paper are considerably better than previously published data on bedmounted ADV meters in natural streams, as reviewed in the paper. Data held by Blake and Packman, which may show better performance, would therefore be a valuable addition to the published literature, although they do not mention any case studies comparable with Pontbren. Since the present authors' paper was submitted, further experiments at a tenth Pontbren site have shown 100\% success rate after calibration, and new data for site SF7 increase its accuracy to 87\%. This further increases the authors' confidence in the Starflow meters and in their method of deployment. The meters performed poorly under low flows, and the advice of Blake and Packman about improving this performance is valid and welcome. As stated in the original paper however, the Pontbren experiment is about flooding and is therefore not designed to measure low flows. Blake and Packman's remark about the suitability of pointing the ADV meters in the downstream direction is well founded in theory (although not supported by the present authors' data), but they neglect to recognise the field circumstances at Pontbren, which required this deployment. In general, their response does not recognise the range of constraints on deployment at Pontbren (which could have been better emphasised in the present authors' original paper). Their implication that the meters were not used carefully at Pontbren reflects their lack of knowledge of these constraints and is inconsistent with the generally high accuracy achieved.

\section{REFERENCES}

ADS (2008) See http://www.adsenv.com/default.aspx?id=73 (accessed 06/08/2008).

Blake JR and Packman JC (2008) Identification and correction of water velocity measurement errors associated with ultrasonic Doppler flow monitoring. Water and Environment Journal. 22(3): 155-167. doi: 10.1111/j.1747-6593.2007.00089.x

Old GH, Leeks GJL, Packman JC, Smith BPG, Lewis S, Goodwin T, Guymer I, Hewitt EJ, Holmes M, Shepherd W and Young A (2002) Fine Sediment Dynamics in Urban Systems: the Bradford Catchment. Centre for Ecology \& Hydrology, Wallingford, UK.

Packman JC and Hewitt EJ (1998) Flood Estimation in Mixed Urban/Rural Catchments, Report to Ministry of Agriculture, Fisheries and Food. Centre for Ecology \& Hydrology, Wallingford, UK.

Packman JC and Hewitt EJ (2000) Urban Runoff Control in the Developing World, Report to Department for International 
Development. Centre for Ecology \&t Hydrology,

Wallingford, UK.

Unidata (2000) STARFLOW Ultrasonic Doppler Instrument User's Manual (Model 6526B), Revision F. Unidata, O'Connor, Australia.

Vermeyen TB (2000) A laboratory evaluation of Unidata's Starflow Doppler flowmeter and MGD Technologies' acoustic Doppler flow meter. Building Partnerships, Proceedings of
Joint Conference on Water Resource Engineering and Water Resources Planning and Management (Hotchkiss RH and Glade M (eds)). American Society of Civil Engineers, Reston, Virginia, p. 318.

Water Cycle Management for new developments (2008) See http://www.wand.uk.net/index.php (accessed 06/08/2008).

Water Research Centre (1987) A Guide to Short Term Flow Surveys of Sewer Systems. WRc, Swindon.

\section{What do you think?}

To discuss this paper, please email up to 500 words to the editor at journals@ice.org.uk. Your contribution will be forwarded to the author(s) for a reply and, if considered appropriate by the editorial panel, will be published as discussion in a future issue of the journal.

Proceedings journals rely entirely on contributions sent in by civil engineering professionals, academics and students. Papers should be 2000-5000 words long (briefing papers should be 1000-2000 words long), with adequate illustrations and references. You can submit your paper online via www.icevirtuallibrary.com/content/journals, where you will also find detailed author guidelines. 